\title{
INVERSE LIMITS WHICH ARE NOT HEREDITARILY INDECOMPOSABLE
}

\author{
ALICE MASON, JOHN J. WALSH AND DAVID C. WILSON
}

\begin{abstract}
Let $X$ be the limit of an inverse sequence $\left\{M_{i}^{n}, f_{i}\right\}$ of closed, connected PL $n$-manifolds with $n>2$. It is shown that if either (1) each $M_{i}^{n}$ is orientable, each $f_{i}$ has nonzero degree, and $\sup _{i}\left\{\operatorname{rank} H_{1}\left(M_{i}^{n}, Z\right)\right\}<\infty$ or (2) $\operatorname{deg}_{z / 2 Z} f_{i} \neq 0$ for each $i$ and $\sup _{i}\left\{\operatorname{rank} H_{1}\left(M_{i}^{n}, \mathbf{Z} / 2 \mathrm{Z}\right)\right\}<\infty$, then $X$ is not hereditarily indecomposable.
\end{abstract}

1. Introduction. An hereditarily indecomposable continuum constructed by R. H. Bing in [1] arises as the inverse limit of 1-spheres with each bonding map having degree 1 . The possibility of extending the construction using inverse sequences of higher dimensional spheres was eliminated by M. Brown [3] who showed that for $n>2$ the inverse limit of $n$-spheres with each bonding map having nonzero degree is not hereditarily indecomposable. A careful examination reveals that Brown's proof can be modified to show that the limit of an inverse sequence of unicoherent $n$-manifolds with the bonding maps having nonzero degree is not hereditarily indecomposable. For manifolds unicoherence is equivalent to the rank of the first homology group (with $\mathbf{Z}$ coefficients) being zero. The purpose of this paper is to improve this result by showing that it suffices to assume that the ranks of the 1st homology groups are uniformly bounded.

MAIN Theorem. Let $X$ be the limit of an inverse sequence $\left\{M_{i}^{n}, f_{i}\right\}$ of closed, connected PL n-manifolds with $n \geqslant 2$.

(a) If each $M_{i}$ is orientable, each $f_{i}$ has nonzero degree, and $\sup _{i}\left\{\operatorname{rank} H_{1}\left(M_{i}, Z\right)\right\}$ $<\infty$, then $X$ is not hereditarily indecomposable.

(b) If $\operatorname{deg}_{\mathrm{z} / 2 \mathrm{z}} f_{i} \neq 0$ for each $i$ and $\sup _{i}\left\{\operatorname{rank} H_{1}\left(M_{i} ; \mathbf{Z} / 2 Z\right)\right\}<\infty$, then $X$ is not hereditarily indecomposable.

The following corollary follows from the Main Lemma in $\$ 4$ and settles a question of Krasinkiewicz [4].

COROllary. For $n>2$ a closed, connected PL, $n$-manifold is not shape equivalent to an hereditarily indecomposable space.

In general, it is necessary to limit the rank of the 1st homology group since the technique used by Bing [2] to construct higher dimensional hereditarily indecomposable continua can be used to produce an example which is the inverse limit of closed 2-manifolds with the bonding maps having degree 1 .

Received by the editors May 2, 1980.

1980 Mathematics Subject Classification. Primary 54F20, 54C56, 54 F55.

Key words and phrases. Inverse limit, hereditarily indecomposable, degree.

(c) 1981 American Mathematical Society 0002-9939/81/0000-0489/\$02.25 
It seems appropriate to recall $M$. Smith's result [5] that finite dimensional cell-like (i.e., limits of inverse sequences of $n$-cells) hereditarily indecomposable spaces are 1-dimensional. Apparently, higher dimensional hereditarily indecomposable spaces are "globally" complicated.

The proof of the main result relies heavily on Brown's techniques in [3] as well as the characterization therein that an inverse limit is hereditarily indecomposable if and only if the bonding maps are "sufficiently crooked". We content ourselves with outlining proofs which are readily found in his article.

2. Preliminaries. Homology and cohomology is singular theory. The integers $\mathbf{Z}$, the field of rationals $Q$, and the field $\mathrm{Z} / 2 \mathrm{Z}$ are the only coefficients that are necessary for this paper. However, for economy of presentation we will state and prove the lemmas in $\$ \S 3$ and 4 with coefficients over an arbitrary field $F$.

Let $f: M^{n} \rightarrow N^{n}$ be a map between closed, connected, $F$-orientable, PL $n$-manifolds. We will say $f$ has nonzero degree over $F$, written $\operatorname{deg}_{F} f \neq 0$, if $f_{*}$ : $H_{n}\left(M^{n} ; F\right) \rightarrow H_{n}\left(N^{n} ; F\right)$ is an isomorphism. Notice that if $f$ is a map between orientable manifolds, then the usual definition of $f$ having nonzero degree (using $\mathbf{Z}$ coefficients) is equivalent to $\operatorname{deg}_{Q} f \neq 0$.

The next condition isolates the important consequence of the assumption that $\operatorname{deg}_{F} f \neq 0$.

If $B \subset N$ is a PL $n$-ball and $X$ is a component of $f^{-1}(B)$, then there is a complementary domain 0 of $X$ so that, for any homotopy $F: M \times I \rightarrow N$ with $F_{0}=f$ and $\left.F\right|_{X \times I}=f$, $F_{1}(0) \supset N-B$.

The point is that if (*) is not true, then $f$ is homotopic to a map $g$, with $g=f$ on $X$, such that $g(0)$ misses a point of $N-B$ for each complementary domain 0 of $X$; since $N-B$ is connected, a further homotopy permits us to assume that the point is the same for each complementary domain and, therefore, that $g(M) \neq N$. Thus, $\operatorname{deg}_{F} g=0$. Since $f$ is homotopic to $g, \operatorname{deg}_{F} f=0$.

\section{Lemmas.}

LEMMA 1. Let $M^{n}$ be a closed, connected, F-orientable, $P L$ manifold and let $C$ be $a$ subpolyhedron of $M^{n}$. If $H^{n-1}(C ; F) \neq 0$ and $i^{*}: H^{n-1}(M ; F) \rightarrow H^{n-1}(C ; F)$ is the zero-map, then $C$ separates $M^{n}$.

Proof. Alexander duality and the exact sequence for the pair $(M, M-C)$ yield

$$
\begin{array}{ccc}
H^{n-1}(M ; F) & \stackrel{i^{*}}{\rightarrow} & H^{n-1}(C ; F) \\
\cong \downarrow & & \cong \downarrow \\
H_{1}(M ; F) & \rightarrow & H_{1}(M, M-C ; F) \rightarrow H_{0}(M-C ; F) \rightarrow F \rightarrow 0
\end{array}
$$

and the square commutes. The hypothesis implies that $H_{1}(M, M-C ; F)$ is nonzero and injects into $H_{0}(M-C ; F)$ and, hence, rank $H_{0}(M-C ; F)>2$. 
Lemma 2. Let $M^{n}$ be a closed, connected, F-orientable, $P L$ manifold. If $P$ is a subpolyhedron which separates points $x$ and $y$ of $M^{n}$ and $i^{*}: H^{n-1}(M ; F) \rightarrow$ $H^{n-1}(C ; F)$ is the zero-map for each component $C$ of $P$, then some component of $P$ separates $x$ and $y$.

Proof. Let $N$ be a regular neighborhood of $P$ chosen not to contain $x$ and $y$; $\partial N$ separates $x$ and $y$. Let $A$ and $B$ be closed connected sets with $M=A \cup B, x \in A$ and $y \in B$, and $A \cap B \subset \partial N$; each of $A$ and $B$ is a union of closures of components of $M-\partial N$. We complete the proof by showing that $A \cap B$ is a single component of $\partial N$.

Write $A \cap B=\cup K_{i}$ where each $K_{i}$ is a component of $\partial N$. Since $A \cap B$ separates $M$ and $\operatorname{Fr} A=F r B=A \cap B$, each $K_{i}$ is a "2-sided" $(n-1)$-dimensional submanifold and, therefore, orientable; in particular, $H^{n-1}\left(K_{i} ; F\right) \simeq F$. The hypothesis implies that each $i^{*}: H^{n-1}(M ; F) \rightarrow H^{n-1}\left(K_{i} ; F\right)$ is the zero-map and Lemma 1 applies to show that each $M-K_{i}$ is disconnected. Since $A$ and $B$ are connected, the only way that this can occur is if $A \cap B$ is a single component of $\partial N$.

Lemma 3. Let $M^{n}$ and $N^{n}$ be closed, connected, F-orientable, PL manifolds with $n \geqslant 2$, let $f: M \rightarrow N$ be a PL map, and let $B \subseteq N$ be a PL $n$-ball such that $f^{-1}(B)$ separates the points $x$ and $y$ in $M$. If $\operatorname{rank} H_{1}(M ; F)=\operatorname{rank} H_{1}(N ; F)$ and $\operatorname{deg}_{F} f \neq$ 0 , then some component of $f^{-1}(B)$ separates $x$ and $y$.

Proof. The strategy is to show that $i^{*}: H^{n-1}(M ; F) \rightarrow H^{n-1}(C ; F)$ is the zero-map for each component $C$ of $f^{-1}(B)$ and, then, to invoke Lemma 2.

Since $\operatorname{deg}_{F} f \neq 0, f_{*}: H_{n}(M ; F) \rightarrow H_{n}(N ; F)$ is an isomorphism and generators $[M] \in H_{n}(M ; F),[N] \in H_{n}(N ; F)$ can be chosen so that $f_{*}([M])=[N]$. Since $f_{*}\left(f^{*}(v) \cap[M]\right)=v \cap f_{*}([M])=v \cap[N]$, the following diagram commutes

$$
\begin{array}{ccc}
H^{n-1}(N ; F) & \stackrel{f^{*}}{\rightarrow} & H^{n-1}(M ; F) \\
\downarrow \cap[N] & & \downarrow \cap[M] \\
H_{1}(N ; F) & \stackrel{\leftarrow}{f_{*}} & H_{1}(M ; F)
\end{array}
$$

where the vertical maps are isomorphisms (Poincaré Duality). It follows that $f_{*}$ is onto and, since rank $H_{1}(N ; F)=\operatorname{rank} H_{1}(M ; F)$, an isomorphism; this forces $f^{*}$ to be an isomorphism. If $C$ is a component of $f^{-1}(B)$, then the latter isomorphism combines with the fact that $H^{n-1}(B ; F)=0$ (this is where we use our assumption that $n \geqslant 2$ ) and the commutative diagram

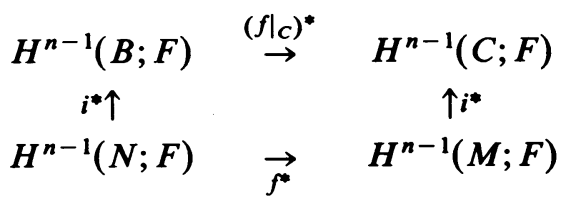

to show that $i^{*}: H^{n-1}(M ; F) \rightarrow H^{n-1}(C ; F)$ is the zero-map. 
LemMa 4. Let $f: M^{n} \rightarrow N^{n}$ be a PL map between closed, connected, F-orientable, $P L$ manifolds where $n \geqslant 2, \operatorname{deg}_{F} f \neq 0$, and $\operatorname{rank} H_{1}(M ; F)=\operatorname{rank} H_{1}(N ; F)$. If $p_{1}$, $p_{2}$, and $p_{3}$ are interior points of pairwise disjoint $P L n$-balls $B_{1}, B_{2}$, and $B_{3}$ contained in $N$, then for some permutation $(i, j, k)$ of $(1,2,3)$ there is a component $X$ of $f^{-1}\left(B_{i}\right)$, a complementary domain 0 of $X$, and points $p_{j}^{\prime}$ and $p_{k}^{\prime}$ in 0 such that

(a) $f\left(p_{j}^{\prime}\right)=p_{j}$,

(b) $f\left(p_{k}^{\prime}\right)=p_{k}$,

(c) $f^{-1}\left(B_{j}\right)$ does not separate $p_{k}^{\prime}$ from $X$, and

(d) $f^{-1}\left(B_{k}\right)$ does not separate $p_{j}^{\prime}$ from $X$.

Outline of Proof (see [3, Lemma 9] for more details). Choose the index $i$, component $X$, and complementary domain 0 so that condition (*) of $\$ 2$ holds and so that if $Y$ is any component of $f^{-1}\left(B_{i}\right)(i=1,2,3)$ in 0 and $\sigma$ is any complementary domain of $Y$ contained in 0 , then (*) does not hold for $0^{\prime}$. Let $j, k$ be any permutation of the remaining integers. Let $C_{1}, \ldots, C_{q}$ be the components of $f^{-1}\left(B_{j}\right)$ contained in 0 which are not separated from $X$ by any other component of $f^{-1}\left(B_{j}\right)$. By Lemma 3, a complementary domain of $\cup C_{i}$ which does not contain $X$ is the complementary domain of some $C_{i}$ and is contained in 0 . Let $W$ be the union of the complementary domains of $\cup C_{i}$ which do not contain $X$. Since each component of $W$ fails to satisfy (*), there is a homotopy $F: M \times I \rightarrow N$ such that $F_{0}=f, F=f$ on $(M-W) \times I$, and $F_{1}(W)$ misses a point of $N-B_{j}$ which we can arrange to be the point $p_{k}$. Since 0 itself satisfies (*), there is a point $p_{k}^{\prime} \in 0$ with $F_{1}\left(p_{k}^{\prime}\right)=p_{k}$. Since $p_{k}^{\prime} \notin W \cup\left(\cup C_{i}\right)$, no $C_{i}$ (and therefore no component of $\left.f^{-1}\left(B_{j}\right)\right)$ separates $p_{k}^{\prime}$ from $X$. Lemma 3 applies to show that $f^{-1}\left(B_{j}\right)$ does not separate $p_{k}^{\prime}$ from $X$. The point $p_{j}^{\prime}$ is found similarly.

4. Proof of Main Theorem. The first reduction is to pass to a subsequence so that the hypothesis of the Main Theorem reads "rank $H_{1}\left(M_{i} ; Q\right)=\operatorname{rank} H_{1}\left(M_{i+1} ; Q\right)$ " in (a) and "rank $H_{1}\left(M_{i} ; Z / 2 Z\right)=\operatorname{rank} H_{1}\left(M_{i+1} ; Z / 2 Z\right)$ " in (b). According to [3, Lemma 3] it suffices to prove the following.

Main Lemma. Let $N^{n}$ be a closed, connected, F-orientable, PL manifold, $n \geqslant 2$. There is an $\varepsilon>0$ such that if $f: M^{n} \rightarrow N^{n}$ is a PL map defined on an F-orientable, closed, connected, $P L$ manifold $M^{n}$ with $\operatorname{rank} H_{1}(M ; F)=\operatorname{rank} H_{1}(N ; F)$ and $\operatorname{deg}_{F} f \neq 0$, then $f$ is not $\varepsilon$-crooked.

Outline of Proof (see [3, Theorem 1] for more details). Adopting the notation of Lemma 4, if $\alpha: I \rightarrow M$ is a path which connects $p_{j}^{\prime}$ to a point in $X$ missing $f^{-1}\left(B_{k}\right)$, "runs in $X$ " for a while if necessary, and then connects a point in $X$ to $p_{k}^{\prime}$ missing $f^{-1}\left(B_{j}\right)$, then $f \circ \alpha$ is not $\varepsilon$-crooked where $2 \varepsilon=\min _{i} d\left(p_{i}, N-B_{i}\right)$.

REMARK. The argument in the second paragraph of the proof of Lemma 3 shows that if $|\operatorname{deg} f| \neq 0$ for a PL map between oriented, closed PL manifolds, then $f_{*}$ : $H_{1}(M ; Q) \rightarrow H_{1}(N ; Q)$ is onto. Combining this fact with the Main Theorem shows that if $X$ is the limit of an inverse sequence of oriented, closed, PL $n$-mainfolds $(n \geqslant 2)$ with the bonding maps having nonzero degree and $X$ is hereditarily indecomposable, then $\check{H}_{1}(X ; Q)$ is infinitely generated. An analogous statement holds for the nonorientable case. 


\section{BIBLIOGRAPHY}

1. R. H. Bing, Concerning hereditarily indecomposable continua, Pacific J. Math. 1 (1951), 43-51.

2. Higher dimensional hereditarily indecompasable continua, Trans. Amer. Math. Soc. 71 (1951), 267-273.

3. M. Brown, On the inverse limit of Euclidean n-spheres, Trans. Amer. Math. Soc. 96 (1960), 129-134.

4. J. Krasinkiewicz, Hereditarily indecomposable representatives of shapes (preprint).

5. M. Smith, Hereditarily indecomposable continua which are inverse limits of n-cubes are tree-like (preprint).

Department of Mathematics, University of Tennessee, Knoxville, Tennessee 37916

Department of Mathematics, University of Florida, Gainesville, Florida 32611 